\title{
HEMATOPOIETIC STEM CELL TRANSPLANTATION FOR PEDIATRIC ACUTE MYELOID LEUKEMIA
}

\author{
Ana Luiza de Melo Rodrigues ${ }^{1,2}$, Victor Gottardello Zecchin ${ }^{3}$, Maria Lúcia M. Lee ${ }^{3}$, Antonella Adriana \\ Zanette ${ }^{1}$, Adriana Seber ${ }^{4}$, Raul Corrêa Ribeiro ${ }^{5}$ \\ 1- Department of Pediatric Oncology and Bone Marrow Transplant, Hospital Erastinho, Curitiba - PR, Brazil, \\ 2- Department of Internal Medicine/Hematology, Federal University of Paraná (UFPR), Curitiba - PR, Brazil \\ 3- Department of Pediatric Oncology and Bone Marrow Transplant, Hospital Beneficência Portuguesa, São Paulo - SP, Brazil \\ 4- Department of Pediatric Oncology and Bone Marrow Transplant, Hospital Samaritano, São Paulo - SP, Brazil \\ 5- Department of Oncology and Global Pediatric Medicine, St. Jude Children's Research Hospital, Memphis - TN, USA
}

Correspondence author: amrodrigues@erastinho.com.br

\begin{abstract}
Acute myeloid leukemia (AML) represents 15\%-20\% of acute leukemias in children, and the risk of treatment failure is based on genetic risk and response to therapy $1-4$. Although the initial remission rate exceeds $90 \%$, more than $30-40 \%$ of children with AML die of refractory/ relapsed disease or treatment-related toxicity5. The best therapeutic results are achieved by integrating intensive chemotherapy, optimal supportive care, and hematopoietic stem cell transplant (HSCT) adapted to each patient's risk of relapse6-9. In 2020, the Brazilian Group for Pediatric Bone Marrow Transplantation of the Brazilian Society of Bone Marrow Transplantation and Cellular Therapy (SBTMO) and the Brazilian Society for Pediatric Oncology (SOBOPE) convened a task force to provide general guidance on HSCT for childhood AML to provide evidence-based guidance for the appropriate management of this disease.
\end{abstract}

Keywords: Hematopoietic Stem Cell Transplantation. Pediatric Acute Myeloid. Leukemia. Clinical Guidelines

Acute myeloid leukemia (AML) represents 15\%-20\% of acute leukemias in children, and the risk of treatment failure is based on genetic risk and response to therapy ${ }^{1-4}$. Although the initial remission rate exceeds $90 \%$, more than $30-40 \%$ of children with AML die of refractory/relapsed disease or treatment-related toxicity ${ }^{5}$. The best therapeutic results are achieved by integrating intensive chemotherapy, optimal supportive care, and hematopoietic stem cell transplant (HSCT) adapted to each patient's risk of relapse ${ }^{6-9}$. In 2020, the Brazilian Group for Pediatric Bone Marrow Transplantation of the Brazilian Society of Bone Marrow Transplantation and Cellular Therapy (SBT$\mathrm{MO})$ and the Brazilian Society for Pediatric Oncology (SOBOPE) convened a task force to review and update the main indications for HSCT for childhood AML based on previous guidelines, intending to provide evidence-based guidance for the appropriate management of this disease.

Currently, HSCT is not recommended for patients in first clinical remission (CR1) when they are classified as low or intermediate risk. Patients classified as high risk, either because of genetic/molecular factors or measurable disease after induction therapies, will be referred for HSCT in CR1.

With the evolution of methods for detecting genetic/ molecular alterations, including the greater availability of gene sequencing techniques, novel genetic alterations have been correlated with different clinical 
and prognostic characteristics. Recent studies have demonstrated new alterations and their clinical, morphological, immunophenotypic and prognostic correlates ${ }^{10}$. The implication of new genetic/molecular markers in $A M L$ is evolving. For example, $A M L$ with KMT2A rearrangements include AML subtypes with with disparate outcomesFor instance, AML cases with $t(6 ; 11)(q 27 ; q 23), t(10 ; 11)(p 12 ; q 23)$ and $\mathrm{t}(10 ; 11)(\mathrm{p} 11.2 ; \mathrm{q} 23)$ have high relapse rates, while patients with $\mathrm{t}(1 ; 11)(\mathrm{q} 21 ; \mathrm{q} 23)$ have an excellent. outcomesThe $t(9 ; 11)(p 12 ; q 23)$ is associated with intermediated risk when occurring in monoblastic or myelomonoblastic leukemia, but a high risk when associated with with acute megakaryoblastic. ${ }^{6}$. The table below show the abnormalities with a more consolidated prognostic impact.

TABLE 1 - Molecular genetic abnormalities with prognostic impact in Pediatric AML

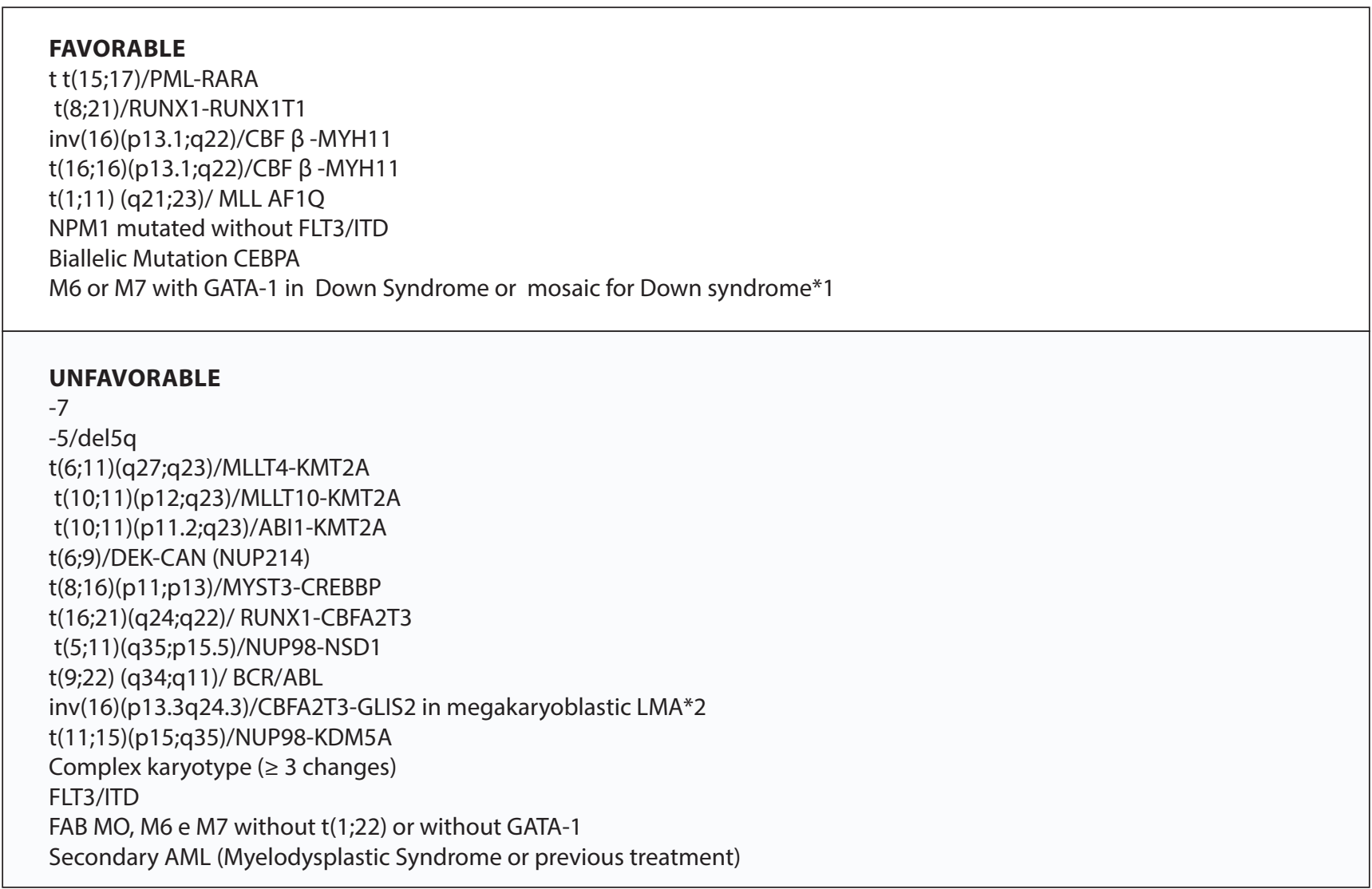

*1 Mast KJ, et al. Pathologic Features of Down Syndrome Myelodysplastic Syndrome and Acute Myeloid Leukemia: A Report From the Children's Oncology Group Protocol AAML0431. Arch Pathol Lab Med. 2020 Apr;144(4):466-472.

*2 Gruber TA, et al. An inv(16)(p13.3q24.3)-encoded CBFA2T3-GLIS2 fusion protein defines an aggressive subtype of pediatric acute megakaryoblastic leukemia*Cancer Cell. (2012) 13; 22(5): 683-697.

In recent years, the measurement of residual disease (MRD) has been incorporated as an additional risk stratifier in the treatment of pediatric AML, usually after the induction cycles. Due to the different methodologies to assess residual disease, the clinical value of MRD is still evolving and should . interpreted within the context of specifictherapeutic protocols.

In countries with limited resources, there is great difficulty in reproducibility and standardization of the methodology used in flow cytometry to quantify low levels of residual disease in AML, which makes interpreting these results and determining their impact on clinical decisions very complex.
Considering the difficulties mentioned above, patients classified as low or intermediate risk, who are referred to HSCT only because they have detectable levels of residual disease after the induction phase, will be evaluated individually. If necessary, the review of MRD tests will be performed by immunophenotyping by the Brazilian Group of Flow Cytometry (GBCFlux) for further definition of the indication of HSCT by the Pediatric Group of SBT$\mathrm{MO}$ and by the Study Group on Acute Myeloid Leukemia (GELMAI) of the Brazilian Society of Pediatric Oncology (SOBOPE)5. 
TABLE 2 - Risk classification based on diagnostic characteristics associated with MRD

\begin{tabular}{l|c}
\hline Low Risk & $\begin{array}{c}\text { Favorable genetic alterations } \\
\text { and } \\
\text { MRD } \leq 1 \% \text { after the first cycle induction }\end{array}$ \\
\hline Intermediate Risk & $\begin{array}{c}\text { Patients who do not have criteria for low or high risk } \\
\text { Unfavorable genetic alterations } \\
\text { or } \\
\text { High Risk }\end{array}$ \\
\hline
\end{tabular}

In relapses, a second remission is attained in about two-thirds of patients with AML; however, lasting remissions in these cases are rare with chemotherapy regimens. Thus, in relapses, allogeneic bone marrow transplantation is always indicated, preferably soon after obtaining a new remission. ${ }^{11,12}$

A recent study reviewing the outcomes of 1940 pediatric AML patients treated with the BFM protocol, from 1987 to 2012, demonstrated that although EFS has remained similar since the 1990s, improvements in supportive care and HSCT have made patients who attained a second remission (CR2) potentially cured, and this resulted in an increase of approximately $20 \%$ in OS in the last 30 years. ${ }^{11}$

In a study with Brazilian HSCT centers for children, adolescents, and young adults, OS and EFS in 4 years were $47 \%$ and $40 \%$, respectively. ${ }^{12}$ Brazilian outcomes of HSCT in children with AML appear to be inferior to those reported in the United States and Europe. A report by Bitan et al. from the Center for International Blood and Marrow Transplant Research (CIBMTR) on 141 pediatric patients with AML who underwent the transplant in CR1 showed a 5-year PFS of 54\% after myeloablative conditioning ${ }^{13}$. Data from the British MRC10 and MRC12 trials showed a 5-year OS of $68 \%$ in children who received marrow transplants from matched sibling donors ${ }^{14}$. The Nordic Society of Paediatric Haematology and Oncology (NOPHO) reported a 3-year EFS of $61 \%$ in children who underwent the transplant in $\mathrm{CR}^{8}$. Locatelli et al. analyzed the outcomes of 243 children with high-risk AML in CR1 who were enrolled in the AIEOP-2002/01 protocol and underwent either allogeneic $(n=141)$ or autologous ( $n=102$ ) HSCT. The 5-year probability of disease-free survival was $73 \%{ }^{15}$. Finally, an AML SCTBFM study aimed at standardizing pediatric HSCT for AML across centers in Germany and Austria reported 4-year EFS and OS of $61 \%$ and $70 \%$, respectively ${ }^{16}$.
The main prognostic factor for the success of HSCT in patients with AML remains the stage of the disease. CIBMTR data show 3-year OS of 70\%, $65 \%$ and $31 \%$, respectively, for patients under 18 years of age undergoing related HSCT in early (CR1), intermediate (CR2) and advanced stages (active disease or $\geq$ CR3 ) of LMA ${ }^{17}$. Patients with treatment-refractory AML or with more than one relapse still have a dismal prognosis ${ }^{18}$.

The results of transplants using related, unrelated (matched or partially matched, with a greater than a 8/10 HLA-match) and haploidentical donors are very similar in $A M L$, with no significant difference between type of donor, whether in overall survival, incidence of acute or chronic graft-versus-host disease (GVHD) ${ }^{12,19}$. In children, bone marrow is preferable in comparison to peripheral blood (PB) as stemcell source, given the higher extensive chronic GVHD and transplant-related mortality with the use of peripheral blood stem cells ${ }^{20,21}$. The use of Umbilical Cord Blood is associated with higher transplant-related mortality in Brazil and should only be used by centers experienced with this stem cell source ${ }^{22}$.

To date, the benefit of autologous marrow transplantation has not been proven when compared to isolated intensive chemotherapy and/or to allogeneic transplantation for non-promyelocytic AML in 1st CR. Thus, autologous transplantation as consolidation should be considered investigational. Conditioning with busulfan area under the curve (AUC) 4000-5000 $\mu$ Mol.min and melphalan total dose (TD) $140 \mathrm{mg} / \mathrm{m} 2$ is currently recommended ${ }^{23-25}$.

As for conditioning in allogeneic transplants, there are better results (toxicity vs relapse) with the use of myeloablative protocols based on busulfan (BU) AUC 4000-5000 $\mu$ Mol.min or based on Total Body Irradiation (TBI) ${ }^{16,26-32}$. 
Although transplantation for active disease $(\geq 5 \%$ blasts in the bone marrow) is controversial, but in cases with adequate performance, benefit from the adapted FLAMSA conditioning scheme has been reported. ${ }^{33-35}$

\section{FLAMSA regimen:}

- Intrathecal chemotherapy D-14

- Etoposide: $150 \mathrm{mg} / \mathrm{m} 2 /$ day, D-13 to D-10

- Fludarabine: $30 \mathrm{mg} / \mathrm{m}^{2} /$ day, D-13 to D-10

- Cytarabine: 2000 mg/m2/day, D-13 to D-10 (4 h after fludarabine)

- Cyclophosphamide: 60 mg/kg/day, D-3 and D-2

- Mesna (1.4 $\mathrm{x}$ dose of cyclophosphamide, divided into 5 doses: $0,3,6,9$ and 12 hours of cyclophosphamide)

- Busulfan $4.8 \mathrm{mg} / \mathrm{kg} /$ day, D-6 and D-5

- If available AUC for busulfan (target 4000-5000 $\mu$ Mol.min), start busulfan one day earlier, then leave one day off the drug, to wait for the result and make necessary adjustments on the day after the break.

- Donor lymphocyte infusion (DLI): D+21 (106 CD3/kg), D+35 (5x106 CD3/kg), D+60 (5x106 $\mathrm{CD} 3 / \mathrm{kg}$ ), start DLI regardless of haematological engraftment, suspend in case of GVHD

- Azacitidine $75 \mathrm{mg} / \mathrm{m} 2 /$ day, for 5 consecutive days, with 1, 2, 3, 4 and 5 months after transplantation (total of 5 cycles)

Due to important differences in the transplant-related mortality rates (MRT) related to age and conditioning regimen, according to the risk/benefit and rates of Event-Free Survival (EFS) and Overall Survival (OS) for patients in pre-HSCT remission, investigators propose different conditioning for children over or under 6 years of age ${ }^{36,37,38}$.

The preparatory regimen will consist of busulfan, cyclophosphamide and melphalan in those six years of age or older. The decision to adopt a preparative regimen containing a combination of three alkylating agents was based on several factors. First, the addition of a third alkylating agent was based on results of a preliminary study by Locatelli et al. ${ }^{37}$, which demonstrated the safety of combining melphalan with busulfan in children, and in the fact that the analysis

A retrospective study of the EWOG-MDS group observed that a conditioning regimen containing a second alkylate was associated with a better EFS and a lower incidence of relapse when compared to regimens employing total body irradiation $(\mathrm{TBI})^{38}$. Strahm published a TRM rate of $21 \%$ in a total cohort of children presenting a "BuCyMel" for advanced myelodysplastic syndromes ${ }^{36}$. Analyzing age groups separately, this MRT was considerably higher in those aged 12 years and over. With the increasing number of AML SCT-BFM 2007 recruitment, an identical MRT pattern has been reported for children and adolescents undergoing transplantation after "BuCyMel" for AML. An MRT of $32 \%$ in patients 12 years of age or older was considered unacceptable, while children under 12 have an excellent result after "BuCyMel," having an MRT rates below $10 \%$. Therefore, we continue to recommend "BuCyMel" for younger children who are eligible for the treatment group.

\section{Description of the "BuCyMel" scheme:}

- Cyclophosphamide: 60 mg/kg/day, D-4 and D-3 (start $24 \mathrm{~h}$ after busulfan)

- Mesna (1.4 x dose of cyclophosphamide, divided into 5 doses: $0,3,6,9$ and 12 hours of cyclophosphamide)

- Melphalan $140 \mathrm{mg} / \mathrm{m}^{2} /$ day D-2

- Busulfan (per kg according to the table 3), D-8, D-7, D-6, D-5

- If available AUC for busulfan (target 4000-5000 $\mu$ Mol.min), start busulfan one day earlier, then leave a day without the drug, to wait for the result and make necessary adjustments the day after the break.

For children over 6 years old, the proposed scheme is the "BuFluMel":

- Fludarabine $30 \mathrm{mg} / \mathrm{m} 2 /$ day from D-7 to D-3

- Busulfan (per kg according to the table 3), D-7, D-6, D-5, D-4

- Melphalan 140 mg/m2/day D-2

- If available AUC for busulfan (target 4000-5000 $\mu$ Mol.min), start busulfan one day earlier, then leave a day without the drug, to wait for the result and make necessary adjustments the day after the break. 
TABLE 3- Busulfan Dosage

\begin{tabular}{|lcc|}
\hline Weight in $\mathbf{~ g g}$ & Busulfan dose $(\mathbf{m g} / \mathbf{k g} /$ day) & Cumulative Dose of Busulfan $\mathbf{( m g} / \mathbf{k g})$ \\
\hline$\leq 9$ & 4,0 & 16,0 \\
\hline$>9-\leq 16$ & 4,8 & 19,2 \\
\hline$>16-\leq 23$ & 4,4 & 17,6 \\
\hline$>23-\leq 34$ & 3,8 & 14,2 \\
\hline$>34$ & 3,2 & 12,8 \\
\hline
\end{tabular}

The recent advent of haploidentical transplantation has made the search for a donor more agile, and as a consequence, has allowed transplants to be carried out for a larger number of patients. According to the exciting results presented by Jaiswal, for transplants with haploidentical donors, the suggested scheme is the one using busulfan AUC 4000-5000 $\mu \mathrm{Mol} . \mathrm{min}$ and melphalan (MEL) total dose (TD) $140 \mathrm{mg} / \mathrm{m} 2$, associated with fludarabine (FLU) TD $150 \mathrm{mg} / \mathrm{m} 2$. The infusion of donor lymphocytes on D+21, D+35 and $\mathrm{D}+60$ had a positive impact on the outcome of patients with advanced disease/worse prognosis.

Depending on the experience of each Transplant Unit, there is the possibility of adopting other conditioning protocols.

\section{About the Graft-versus-Host Disease (GVHD) prophylaxis regimen:}

- In HLA-matched sibling donor (MSD) allo-HSCT, calcineurin inhibitors (Cyclosporine - CSP 2mg/ $\mathrm{kg}$ or Tacrolimus -TAC $0.05 \mathrm{mg} / \mathrm{kg}$ in two divided I.V. doses a day) as a single agent should be started on D-1, and switched to their corresponding P.O. formulations, with strict dose adjustment based on serum levels $(100-200 \mathrm{mcg} / \mathrm{L}$ for CSP and $5-15 \mathrm{ng} / \mathrm{ml}$ for TAC), until 3 months after transplant, with subsequent tapering, in the absence of graft-versus-host disease (GVHD) ${ }^{40-42}$.

- In HLA-matched unrelated donor (MUD) HSCT, calcineurin inhibitors (Cyclosporine - CSP 2mg/ $\mathrm{kg}$ or Tacrolimus - TAC $0.05 \mathrm{mg} / \mathrm{kg}$ in two divided I.V. doses a day) as a single agent should be started on D-1, and switched to their corresponding P.O. formulations, with strict dose adjustment based on serum levels $(100-200 \mathrm{mcg} / \mathrm{L}$ for CSP and $5-15 \mathrm{ng} / \mathrm{ml}$ for TAC), until 3 months after transplant, with subsequent tapering, in the absence of graft-versus-host disease (GVHD) ${ }^{40,42}$.

*The use of single-agent, post-transplant cyclophosphamide (PTCy) at a dose of $50 \mathrm{mg} / \mathrm{kg}$ two days between $D+3$ and $D+4$ has shown similar results regarding GVHD control, although further studies are awaited in order to define the optimal regimen in terms of long-term outcome for these patients ${ }^{43-45}$.

- In unrelated allo-HSCT, CSP (at the same dose as that for related donor transplants) combined with methotrexate (MTX) for a short period of time (i..e, on days $+1,+3,+6$ and +11 ) is the standard prophylactic regimen. MTX is used at an initial dose of $15 \mathrm{mg} / \mathrm{m}^{2}$, followed by three doses of $10 \mathrm{mg} / \mathrm{m}^{2}$, TAC at a total daily dose of $0.05 \mathrm{mg} /$ $\mathrm{kg}$ can also be used, with similar results ${ }^{40,42}$. In contrast, the combination of mycophenolate mofetil (MMF) with CSP was shown to be less effective ${ }^{43-45}$.

- Although the use of anti-thymocyte globulin (ATG), primarily for the prevention of GVHD, has been consolidated in unrelated donor HSCT in adults, there is limited evidence as to its benefit in the pediatric population, even though it is used in most protocols. In a randomized study comparing different dose regimens of ATG, use of ATG at lower doses $(4,5-6 \mathrm{mg} / \mathrm{kg})$ could reduce the rate of infection while maintaining similar acute and chronic GVHD rates, as well as relapse rates. The investigators concluded that low-dose ATG should be the standard serotherapy regimen for URD HCST in children with hematologic malignancies ${ }^{46}$, even though it should be borne in mind that the different ATG formulations available have variable immune responses, which may hinder any definitive conclusions as to its real benefit in this regard.

- In haploidentical HSCT, cyclophosphamide is generally used at a dose of $50 \mathrm{mg} / \mathrm{kg} /$ day, in a 2-hour infusion, on D+3 and D+4, coupled with mesna $(100-160 \%$ of the cyclophosphamide dose), in combination with a calcineurin inhibitor (CSP or TAC) and MMF $(15 \mathrm{mg} / \mathrm{kg} /$ dose q8h; maximum dose $2 \mathrm{~g} /$ day), both starting on $\mathrm{D}+5$. Both these immunosuppressants are usually kept for 3 months post-transplant ${ }^{47,48}$. 
As for UCB transplantation, the immunosuppressive regimen usually comprises the combination of a calcineurin inhibitor with MMF. Studies on the association of CSP with low-dose MTX or with corticosteroids have yielded worse results, as well as a greater graft failure rate ${ }^{42}$.

\section{Best time points for MRD assessment:}

Pre-HSCT: MRD assessments should be made immediately before allo-HSCT.

Post-HSCT: MRD assessments by multiparameter flow cytometry (MFC) and/or reverse transcription quantitative polymerase chain reaction (RT-qPCR) are accurate in predicting relapse at days $+30,+60$, +90 , and +180 post-HSCT.

Any detectable MRD level on days +180 and +365 post-HSCT is highly predictive of relapse and poor survival ${ }^{49}$. When decisions that may change patient management are based on low levels of MRD, we would recommend that the SBTMO - MRD Working Group GBFLUX may review the flow cytometric data to increase accuracy of the results.

Despite the immunological effect of the grafted cells against leukemia, the toxicity and mortality related to the procedure remain large barriers. The heterogeneity of data related to patient selection, type of conditioning for HSCT and donors makes data interpretation difficult in the pediatric population, particularly in developing countries, but procedure-related mortality is estimated to be between $10-25 \%$ in our country ${ }^{12}$.

\section{REFERENCES}

1. Estey $\mathrm{E}$, Dohner $\mathrm{H}$. Acute myeloid leukaemia. Lancet. 2006;368(9550):1894-1907.

2. Lagunas-Rangel FA, Chavez-Valencia V, Gomez-Guijosa MA, Cortes-Penagos C. Acute myeloid leukemia-genetic alterations and their clinical prognosis. Int J Hematol Oncol Stem Cell Res. 2017;11(4):328-339.

3. Sadeghian MH, Rezaei Dezaki Z. Prognostic value of EVI1 expression in pediatric acute myeloid leukemia: a systematic review. Iran J Pathol. 2018;13(3):294-300.

4. Rubnitz JE. Current management of childhood acute myeloid leukemia. Paediatr Drugs. 2017;19(1):1-10.
Another key point for better results is carrying out the transplant without delay, which is hampered by the scarcity of beds for patients dependent on the public health system. Patients in first and second remissions are potentially curable with HSCT, but from the second relapse and/or when the patient has active disease, there is a drastic reduction in the chances of cure. Delaying the procedure is harmful both due to the risk of losing the remission status as well as exposure to the toxicity of a new cycle of chemotherapy, which can worsen the child's performance for transplantation, or even be fatal ${ }^{12}$.

We recommend in the AML the HLA typing of the patient, parents and siblings at diagnosis. If no related donor is identified, collect the patient's anti-HLA antibody test and start search for a donor at REDOME.

Once the indication for transplantation is confirmed, the interaction between the pediatric oncologist and the transplant center is essential for the prompt donor search and planning of the procedure.

Currently, advances have been achieved, in particular through the connection between the Brazilian Societies of Bone Marrow Transplant - SBTMO, of the Pediatric Oncology Society - SOBOPE, theFlow Cytometry - GBFlux and the Brazilian Association of Hematology, Hemotherapy and Cell Therapy - $\mathrm{ABHH}$, in the challenging goal of improving the treatment of children and adolescents with AML. These efforts will also contribute to agreater knowledge of Brazilian experience.

5. Burke MJ, Wagner JE, Cao Q, et al. Allogeneic hematopoietic cell transplantation in first remission abrogates poor outcomes associated with high-risk pediatric acute myeloid leukemia. Biol Blood Marrow Transplant. 2013;19(7):10211025.

6. Rubnitz JE. How I treat pediatric acute myeloid leukemia. Blood. 2012;119(25):5980-5988.

7. Gibson BE, Webb DK, Howman AJ, et al. Results of a randomized trial in children with acute myeloid leukaemia: medical research council AML12 trial. Br J Haematol. 2011;155(3):366-376.

8. Abrahamsson J, Forestier E, Heldrup J, et al. Response- guided induction therapy in pediatric 
acute myeloid leukemia with excellent remission rate. J Clin Oncol. 2011;29(3): 310-315.

9. Rasche M, Zimmermann M, Borschel L, et al. Successes and challenges in the treat- ment of pediatric acute myeloid leukemia: a retrospective analysis of the AML-BFM trials from 1987 to 2012. Leukemia. 2018;32(10):2167-2177.

10. Shiba, Norio, et al. "Transcriptome analysis offers a comprehensive illustration of the genetic background of pediatric acute myeloid leukemia." Blood advances 3.20 (2019): 3157-3169.

11. Rasche M, Zimmermann $M$, Borschel $L$, et al. Successes and challenges in the treatment of pediatric acute myeloid leukemia: a retrospective analysis of the AML-BFM trials from 1987 to 2012. Leukemia. 2018;32(10):2167-2177.

12. de Melo Rodrigues, Ana Luiza, et al. Allogeneic Hematopoietic Stem Cell Transplantation for Children and Adolescents with Acute Myeloid Leukemia in Brazil: A Multicentric Retrospective Study. Cell Transplantation. 2020;29:0963689720949175.

13. Bitan M, He W, Zhang MJ, et al. Transplantation for children with acute myeloid leukemia: a comparison of outcomes with reduced intensity and myeloablative regimens. Blood. 2014;123(10):1615-1620.

14. Gibson BE, Wheatley K, Hann IM, et al. Treatment strategy and long-term results in paediatric patients treated in consecutive UK AML trials. Leukemia. 2005;19(12):2130-2138.

15. Locatelli $F$, Masetti $R$, Rondelli $R$, et al. Outcome of children with high-risk acute myeloid leukemia given autologous or allogeneic hematopoietic cell transplantation in the aieop AML-2002/01 study. Bone Marrow Transplant. 2015;50(2):181-188.

16. Sauer MG, Lang PJ, AlbertMH, et al. Hematopoietic stem cell transplantation for children with acute myeloid leukemia-results of the AML SCTBFM 2007 trial. Leukemia. 2020;34(2):613-624.

17. D'Souza A, Fretham C, Lee SJ, Arora M, Brunner J, Chhabra S, et al. Current Use of and Trends in Hematopoietic Cell Transplantation in the United States. Biology of Blood and Marrow Transplantation. 2020;26(8):e177-e82.

18. Golub TR, Arceci RJ. Acute myelogenous leukemia. In: Pizzo PA, Poplack DG, editors. Principles and Practice of Pediatric Oncology. Philadelphia: Lippincot; 2002. p. 545-89.

19. Leung, Wing, et al. "High success rate of hematopoietic cell transplantation regardless of donor source in children with very high-risk leukemia." Blood 118.2 (2011): 223-230.

20. Peters C, Schrappe $M$, von Stackelberg $A$, Schrauder $A$, et al. Stem-cell transplantation in children with acute lymphoblastic leukemia: a prospective international multicenter trial comparing sibling donors with matched unrelated donors-The ALL-SCT-BFM-2003 Trial. J Clin Oncol. 2015 Apr 10;33(11):1265-74

21. Burke MJ, Verneris MR, Le Rademacher J, et al. Transplant Outcomes for Children with T Cell Acute Lymphoblastic Leukemia in Second Remission: A Report from the Center for International Blood and Marrow Transplant Research. Biol Blood Marrow Transplant. 2015;21(12):21542159.

22. Tavares RCB, Bonfim CS, Seber A, Pereira Lermontov S, Coulturato V, Zecchin VG, Ribeiro L, Fernandes JF, Daudt LE, Grecco CS, Darrigo-Jr LG, Villela N, Nichele S, Gouveia R, Bouzas LF, Hamerschlak N, Vigorito AC, da Silva PM, da Silva PO, da Silva CC, de Souza Fernandez C, Flowers ME, Arcuri LJ. Hematopoietic cell transplantation in pediatric patients with acute leukemias or myelodysplastic syndrome using unrelated adult or umbilical cord blood donors in Brazil. Pediatr Transplant. 2020 Nov;24(7):e13789. doi: 10.1111/petr.13789. Epub 2020 Aug 5. PMID: 32757316

23. Yamamoto, Shohei, et al. "Hematopoietic stem cell transplantation for pediatric acute promyelocytic leukemia in Japan." Pediatric Blood \& Cancer 67.5 (2020): e28181.

24. Dvorak, Christopher C., et al. "Hematopoietic stem cell transplant for pediatric acute promyelocytic leukemia." Biology of Blood and Marrow Transplantation 14.7 (2008): 824-830.

25. Woods, William G., et al. "A comparison of allogeneic bone marrow transplantation, autologous bone marrow transplantation, and aggressive chemotherapy in children with acute myeloid leukemia in remission: a report from the Children's Cancer Group." Blood, The Journal of the American Society of Hematology 97.1 (2001): 56-62. 
26. Michel, G., et al. "Allogeneic bone marrow transplantation for children with acute myeloblastic leukemia in first complete remission: impact of conditioning regimen without total-body irradiation--a report from the Soci $\sqrt{ } \odot \mathrm{t} \sqrt{ } \odot$ Fran $\sqrt{ }$ ßaise de Greffe de Moelle." Journal of clinical oncology 12.6 (1994): 1217-1222.

27. Pession, Andrea, et al. "Results of the AIEOP AML 2002/01 multicenter prospective trial for the treatment of children with acute myeloid leukemia." Blood, The Journal of the American Society of Hematology 122.2 (2013): 170-178.

28. Solomon, Scott R., et al. "TBI-based Myeloablative Haploidentical stem cell transplantation is a safe and effective alternative to unrelated donor transplantation in patients without matched sibling donors." (2014): 426-426.

29. Small, Trudy N., et al. "Intravenous busulfan and melphalan, tacrolimus, and short-course methotrexate followed by unmodified HLAmatched related or unrelated hematopoietic stem cell transplantation for the treatment of advanced hematologic malignancies." Biology of Blood and Marrow Transplantation 13.2 (2007): 235-244.

30. De Berranger, E., et al. "Impact on long-term OS of conditioning regimen in allogeneic BMT for children with $A M L$ in first $C R$ : $T B I+C Y$ versus $B U+C Y$ : a report from the Société Française de Greffe de Moelle et de Thérapie Cellulaire." Bone marrow transplantation 49.3 (2014): 382-388.

31. Kobos, Rachel, et al. "Allogeneic hematopoietic stem cell transplantation for pediatric patients with treatment-related myelodysplastic syndrome or acute myelogenous leukemia." Biology of Blood and Marrow Transplantation 18.3 (2012): 473-480.

32. Beier, R., et al. "Allo-SCT using BU, CY and melphalan for children with AML in second CR."Bone marrow transplantation 48.5 (2013): 651-656.

33. Kolb, Hans-Jochem, and Christoph Schmid. "The FLAMSA concept-past and future." Annals of Hematology (2020): 1-10.

34. Owattanapanich, Weerapat, et al. "FLAMSA-RIC for stem cell transplantation in patients with acute myeloid leukemia and myelodysplastic syndromes: a systematic review and meta-analysis." Journal of clinical medicine 8.9 (2019): 1437.
35. Holtick, Udo, et al. "FLAMSA reduced-intensity conditioning is equally effective in AML patients with primary induction failure as well as in first or second complete remission." European journal of haematology 96.5 (2016): 475-482.

36. Strahm, Brigitte, et al. "Hematopoietic stem cell transplantation for advanced myelodysplastic syndrome in children: results of the EWOG-MDS 98 study." Leukemia 25.3 (2011): 455-462.

37. Locatelli, Franco, et al. "Role of allogeneic bone marrow transplantation from an HLAidentical sibling or a matched unrelated donor in the treatment of children with juvenile chronic myeloid leukaemia." British journal of haematology 92.1 (1996): 49-54.

38. Locatelli, Franco, et al. "Allogeneic bone marrow transplantation for chronic myelomonocytic leukemia in childhood: a report from the European Working Group on Myelodysplastic.

39. Jaiswal, Sarita Rani, et al. "Improved Outcome of Refractory/Relapsed Acute Myeloid Leukemia after Post-Transplantation Cyclophosphamide-Based Haploidentical Transplantation with Myeloablative Conditioning and Early Prophylactic Granulocyte Colony-Stimulating Factor-Mobilized Donor Lymphocyte Infusions." Biology of Blood and Marrow Transplantation 22.10 (2016): 1867-1873.

40. Lv X, Qi J, Zhou M, et al. Comparative efficacy of 20 graft-versus-host disease prophylaxis therapies for patients after hematopoietic stem-cell transplantation: A multiple-treatments network meta-analysis. Crit Rev Oncol Hematol. 2020 Jun; 150:102944.

41. Elgarten CW, Arnold DE, Bunin NJ, Seif AE. Outcomes of matched sibling donor bone marrow transplantation in children using single-agent calcineurin inhibitors as prophylaxis for graft versus host disease. Pediatr Blood Cancer. 2018;65(1): 10.1002/pbc.26726.

42. Lawitschka A, Lucchini G, Strahm B, et al. Pediatric acute graft-versus-host disease prophylaxis and treatment: surveyed real-life approach reveals dissimilarities compared to published recommendations. European Society for Blood, Marrow Transplantation (EBMT) Pediatric Diseases Working Party. Transpl Int. 2020 Jul;33(7):762-772. 
43. Jacoby E, Chen A, Loeb DM, Gamper CJ, Zambidis E, Llosa NJ, Huo J, Cooke KR, Jones R, Fuchs E, Luznik L, Symons HJ. Single-Agent Post-Transplantation Cyclophosphamide as Graft-versusHost Disease Prophylaxis after Human Leukocyte Antigen-Matched Related Bone Marrow Transplantation for Pediatric and Young Adult Patients with Hematologic Malignancies. Biol Blood Marrow Transplant. 2016 Jan;22(1):112-8.

44. Kwon M, Bail $\sqrt{ } \bigcirc \mathrm{n} R$, Pascual-Casc $\sqrt{ } \geq n$ MJ, Gallardo-Morillo Al, Garc $\sqrt{\neq}$ a Sola A, Balsalobre P, Sol $\sqrt{ }{ }^{\circ} \mathrm{L}$, Dorado $N, \mathrm{Mu} \sqrt{ } \pm$ oz $C$, Serrano $D$, Mart $\sqrt{ } \neq$ nez-Laperche $C, B u \sqrt{ \pm 0}$ I, Anguita J, $\mathrm{D} \sqrt{ } \neq$ ez-Martin JL. Posttransplant cyclophosphamide vs cyclosporin A and methotrexate as GVHD prophylaxis in matched sibling transplantation. Blood Adv. 2019 Nov 12;3(21):3351-3359.

45. Yerushalmi R, Shem-Tov N, Danylesko I, Shouval $R$, Nagler A, Shimoni A. The combination of cyclosporine and mycophenolate mofetil is less effective than cyclosporine and methotrexate in the prevention of acute graft-versus host disease after stem-cell transplantation from unrelated donors.Am J Hematol. 2017 Mar;92(3):259-268.

46. Locatelli F, Bernardo ME, Bertaina A, Rognoni C, Comoli P, Rovelli A, et al. Efficacy of two different doses of rabbit anti-lymphocyte globulin to prevent graft-versus-host disease in children with haematological malignancies transplanted from an unrelated donor: a multicentre, ran- domised, open-label, phase 3 trial. Lancet Oncol. 2017;18(8):1126-36. https://doi.org/10.1016/ S1470-2045(17)30417-5 Multicenter randomized-controlled study demonstrating that a lower dose of ATLG determines a better outcome as compared to the use of an higher dose. This was due to a lower incidence of infectious complications, while the incidence of acute and chronic GvHD, as well as that of recurrence of the original disease was not significantly affected.

47. Annalisa Ruggeri, Jacques-Emmanuel Galimard, Olesya Paina, Franca Fagioli, Abdelghani Tbakhi, Akif Yesilipek, Jos $\sqrt{ } \odot$ Maria Fernandez Navarro, Maura Faraci, Rose-Marie Hamladji, Elena Skorobogatova, Amal Al-Seraihy, Mikael Sundin, Concepcion Herrera, Jose Rif $\sqrt{ } \geq n$, Arnaud Dalissier, Franco Locatelli, Vanderson Rocha, Selim Corbacioglu, Outcomes of Unmanipulated Haploidentical Transplantation Using Post-Transplant Cyclophosphamide (PT-Cy) in Pediatric Patients With Acute Lymphoblastic Leukemia. Transplantation and Cellular Therapy, 2021; 27(5): 424.e1-424.e9

48. Shah, R.M. Contemporary haploidentical stem cell transplant strategies in children with hematological malignancies. Bone Marrow Transplant. 2021; 56, 1518-1534.

49. Nagler, Arnon, et al. "Measurable residual disease (MRD) testing for acute leukemia in EBMT transplant centers: a survey on behalf of the ALWP of the EBMT." Bone Marrow Transplantation 56.1 (2021): 218-224. 